\title{
Mechanical Proof of the Maxwell Speed Distribution
}

\author{
Tsung-Wu Lin ${ }^{1} \&$ Hejie Lin $^{2}$ \\ ${ }^{1}$ Department of Civil Engineering, National Taiwan University, Taipei, Taiwan \\ ${ }^{2}$ Department of Mechanical Engineering, Oakland University, Rochester Hills, Michigan, USA \\ Correspondence: Tsung-Wu Lin, Department of Civil Engineering, National Taiwan University, Taipei, Taiwan. E-mail: \\ twlin@ntu.edu.tw
}

Received: December 26, 2018 Accepted: January 18, 2019 Online Published: January 25, 2019

doi:10.5539/ijsp.v8n2p90

URL: https://doi.org/10.5539/ijsp.v8n2p90

\begin{abstract}
This article derives the probability density function $\psi\left(\xi ; \mathrm{x}, \mathrm{x}^{\prime}\right)$ of the resulting speed $\xi$ from the collision of two particles with speeds $\mathrm{x}$ and $\mathrm{x}^{\prime}$. This function had been left unsolved for about 150 years. Then uses two approaches to obtain the Maxwell speed distribution: (1) Numerical iteration: using the equation

$$
P_{\text {new }}(\xi)=\int_{0}^{\infty} \int_{0}^{\infty} \psi\left(\xi ; \mathrm{x}, \mathrm{x}^{\prime}\right) \cdot P_{\text {old }}(\mathrm{x}) \cdot P_{\text {old }}\left(\mathrm{x}^{\prime}\right) d x \mathrm{dx}^{\prime}
$$

to get the new speed distribution from the old speed distribution. Also, after 9 iterations, the distribution converges to the Maxwell speed distribution. (2) Analytical integration: using the Maxwell speed distribution as the $P_{\text {old }}(\mathrm{x})$, and then getting $P_{\text {new }}(\xi)$ from the above integration. The result of $P_{\text {new }}(\xi)$ from analytical integration is proved to be exactly the Maxwell speed distribution.
\end{abstract}

Keywords: Maxwell speed distribution, Maxwell-Boltzmann distribution, collision of particles, kinetic theory of gases

\section{Overview}

Maxwell first provided the Maxwell speed distribution in 1860 on statistical heuristic bases (Maxwell, 1860a,b). Maxwell in 1867 (Maxwell) and Boltzmann in 1872 (Boltzmann) carried out some more investigations into the physical meaning of the distribution. The simplest way to prove the Maxwell speed distribution is from the statistical view: beginning from the Boltzmann distribution of energy state which is proportional to the square of velocity, and extending to three velocities in three directions and summing the same speed distribution in all three directions to get the Maxwell speed distribution (Brush, 1966, Landau et al., 1969, McQuarrie, 1976, Garrod, 1995, Maudlin, 2013). Therefore, the distribution is also known as the Maxwell-Boltzmann distribution. The standard speed distribution function is listed as follows along with a more compacted parameter $h$ which is the inverse of the most probable speed $v_{m p}$, i.e., $v_{m p}=h^{-1}$.

$$
P(v)=\frac{4}{\sqrt{\pi}}\left(\frac{m}{2 k T}\right)^{3 / 2} v^{2} e^{\left(\frac{-m v^{2}}{2 k T}\right)}=\frac{4 h^{3}}{\sqrt{\pi}} v^{2} e^{-h^{2} v^{2}}
$$

where $k$ is the Boltzmann constant, $T$ is the equilibrium temperature, $m$ is the particle mass, and $h=\sqrt{\frac{m}{2 k T}}$. In 1872, Boltzmann gave the following equation:

$$
\mathrm{dn}=\mathrm{f}(\mathrm{x}, \mathrm{t}) \mathrm{dx} \cdot \mathrm{f}\left(\mathrm{x}^{\prime}, \mathrm{t}\right) \mathrm{dx} \cdot \psi\left(\xi ; \mathrm{x}, \mathrm{x}^{\prime}\right) \mathrm{d} \xi
$$

where $f(x, t) d x$ is the number of particles with speed between $x$ and $x+d x$, and similarly for $f\left(x^{\prime}, t\right) d x^{\prime}, d n$ is the number of particles with speed between $\xi$ and $\xi+d \xi$. If we let $f(\xi, t+d t)=d n / d \xi$, and rewrite Eq.(1) as

$$
\mathrm{f}(\xi, \mathrm{t}+\mathrm{dt})=\int_{0}^{\infty} \int_{0}^{\infty} \psi\left(\xi ; \mathrm{x}, \mathrm{x}^{\prime}\right) \cdot \mathrm{f}(\mathrm{x}, \mathrm{t}) \cdot \mathrm{f}\left(\mathrm{x}^{\prime}, \mathrm{t}\right) d x \mathrm{dx}^{\prime}
$$

As $t \rightarrow \infty, \mathrm{f}(\mathrm{x}, \mathrm{t}) \rightarrow P(x)$, the correct distribution, $P(x)$, should satisfy the following new integral equation

$$
\mathrm{P}(\xi)=\int_{0}^{\infty} \int_{0}^{\infty} \psi\left(\xi ; \mathrm{x}, \mathrm{x}^{\prime}\right) \cdot \mathrm{P}(\mathrm{x}) \cdot \mathrm{P}\left(\mathrm{x}^{\prime}\right) d x \mathrm{dx^{ \prime }}
$$

Boltzmann said that "Since this calculation $\left(\psi\left(\xi ; \mathrm{x}, \mathrm{x}^{\prime}\right)\right.$, add by authors), although tedious, is not at all difficult, ...". However, until now, this calculation is still missing in the literature. As shown in Section 2, the function can be derived based on Newton's laws of motion, and therefore it is also a mechanical proof of the Maxwell speed distribution. 
After we get the function $\psi\left(\xi ; x, x^{\prime}\right)$, we use two approaches to get the Maxwell speed distribution: (1) Numerical iteration: using the following equation to get the new distribution from the old one. Also, found that the final distribution after 9 iterations converges to the Maxwell speed distribution as shown in Section 3.

$$
P_{\text {new }}(\xi)=\int_{0}^{\infty} \int_{0}^{\infty} \psi\left(\xi ; \mathrm{x}, \mathrm{x}^{\prime}\right) \cdot P_{\text {old }}(\mathrm{x}) \cdot P_{\text {old }}\left(\mathrm{x}^{\prime}\right) d x \mathrm{dx}{ }^{\prime}
$$

(2) Analytical integration: using the Maxwell speed distribution as $P_{\text {old }}$ to get $P_{\text {new }}$ from integration. And the $P_{\text {new }}$ from analytical integration is exactly the Maxwell speed distribution as shown in Section 4.

\section{Derivation of $\psi\left(\xi ; x, x^{\prime}\right)$}

Before processing to derive the function $\psi\left(\xi ; \mathrm{x}, \mathrm{x}^{\prime}\right)$, we change the variables $\xi$ to $v, \mathrm{x}$ to $v_{j}$ and $\mathrm{x}^{\prime}$ to $v_{k}$ and rewrite the function as $\psi\left(v ; v_{j}, v_{k}\right)$. For ease of reference, the resulting function is listed as follows. Since $v_{j}$ and $v_{k}$ are exchangeable, only the functions for $v_{j} \geq v_{k}$ are listed.

$$
\begin{aligned}
& \psi\left(v ; v_{j}, v_{k}\right)=\frac{v}{v_{j} v_{k}} \sin ^{-1}\left(\frac{2 v}{v_{j}^{2}+v_{k}^{2}} \sqrt{v_{j}^{2}+v_{k}^{2}-v^{2}}\right), \quad 0 \leq v \leq v_{k} \text { or } \quad v_{j} \leq v \leq \sqrt{v_{j}^{2}+v_{k}^{2}} \\
& =\frac{v}{v_{j} v_{k}} \sin ^{-1}\left(\frac{2 v_{j} v_{k}}{v_{j}^{2}+v_{k}^{2}}\right), \quad 0 \leq v_{k} \leq v \leq v_{j} \\
& =0 \text {, } \\
& v \geq \sqrt{v_{j}^{2}+v_{k}^{2}}
\end{aligned}
$$

\subsection{For Special Case of $v_{k}=0$}

Let $\mathrm{v}_{0}=v_{j}$ be the speed of particle 1 with mass $\mathrm{M}_{1}$ which will hit particle 2 with mass $\mathrm{M}_{2}$ at rest. After a collision, the new particle speeds are $v_{1}$ and $v_{2}$ as shown in Fig. 1.

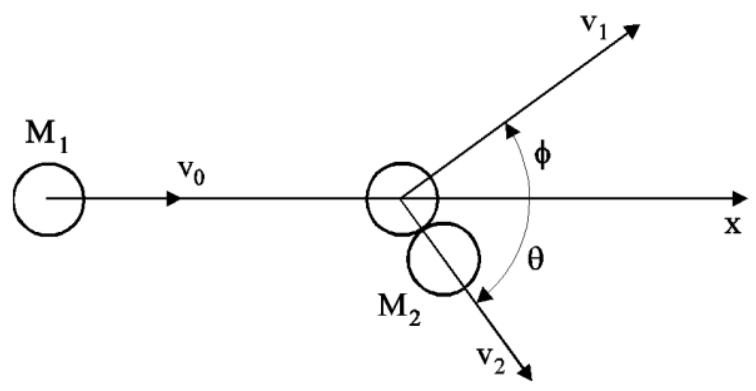

Figure 1. The collision of two particles where particle 2 is at rest

Based on Newton's laws of motion, the total momenta before and after the collision are the same (Eqs.(6-7)). Also, for elastic collision, the total energies before and after the collision are also the same (Eq.(8)).

For $\mathrm{M}_{1}=\mathrm{M}_{2}$, we get the solutions as (Note 1)

$$
\begin{gathered}
\mathrm{M}_{1} \mathrm{v}_{0}=\mathrm{M}_{2} \mathrm{v}_{2} \cos \theta+\mathrm{M}_{1} \mathrm{v}_{1} \cos \phi \\
0=\mathrm{M}_{2} \mathrm{v}_{2} \sin \theta-\mathrm{M}_{1} \mathrm{v}_{1} \sin \phi \\
\mathrm{M}_{1} \mathrm{v}_{0}^{2} / 2=\mathrm{M}_{2} \mathrm{v}_{2}^{2} / 2+\mathrm{M}_{1} \mathrm{v}_{1}^{2} / 2
\end{gathered}
$$

$$
\begin{gathered}
\mathrm{v}_{2}=\mathrm{v}_{0} \cos \theta \\
\mathrm{v}_{1}=\mathrm{v}_{0} \sin \theta \\
\sin (\theta+\phi)=1 \text { or } \phi=\frac{\pi}{2}-\theta
\end{gathered}
$$

The solutions can be represented as Fig. 2, where $\mathrm{v}_{1}=\overline{B P}$, and $\mathrm{v}_{2}=\overline{B Q}$. Note that, after the collision, $\mathrm{P}$ and $\mathrm{Q}$ are always located on the sphere surface and the probability is uniform on this surface. Since the probability of the point inside the circle in Fig. 2(b) (radius=diameter of a particle) is uniformly distributed (Note 2), we get the probability of $\mathrm{Q}$ located between $\theta$ and $\theta+d \theta$ as $\mathrm{P}_{\theta}(\theta) d \theta=2 \pi\left(\mathrm{v}_{0} \cos \theta \sin \theta\right)\left(\mathrm{v}_{0} d \theta\right) /\left(\pi \mathrm{v}_{0}^{2}\right)$. And change the variable from $\theta$ to $v=\mathrm{v}_{0} \cos \theta$ by $\mathrm{P}_{v}(v)=\mathrm{P}_{\theta}(\theta)\left|\frac{d \theta}{d v}\right|$ to get

$$
\mathrm{P}_{v}(v)=\frac{2 v}{\mathrm{v}_{0}^{2}}, \quad \text { for } 0 \leq v \leq \mathrm{v}_{0} ; \text { and } \mathrm{P}_{v}(v)=0 \text {, otherwise }
$$




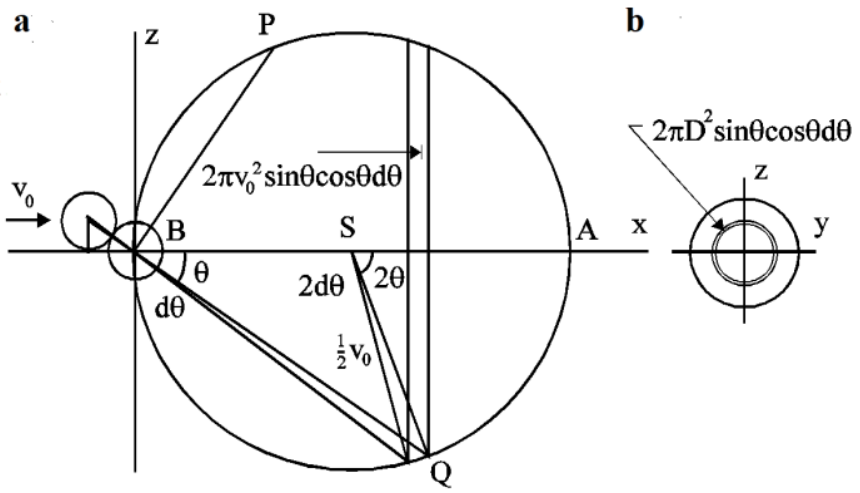

Figure 2. The relation between collision point as shown in (b) and new velocity $\overline{\mathrm{BQ}}$ in (a)

\subsection{For General Case of $v_{j} \geq v_{k}>0$}

Let $v_{j}=\left|\bar{v}_{1}\right|$ and $v_{k}=\left|\bar{v}_{2}\right|$ be the speeds of two particles before a collision. After the collision, the new particle speeds are $v_{i 1}=\left|\tilde{v}_{1}\right|$ and $v_{i 2}=\left|\tilde{v}_{2}\right|$. Let $\mathrm{v}_{0}=\overline{\mathrm{v}}_{1}-\overline{\mathrm{v}}_{2}$, and follow the same procedures of Section 2.1 to get $\mathrm{v}_{1}$ and $v_{2}$, and therefore to get $\tilde{v}_{1}=\bar{v}_{2}+v_{1}$ and $\tilde{v}_{2}=\bar{v}_{2}+v_{2}$ as shown in Fig. 3 .

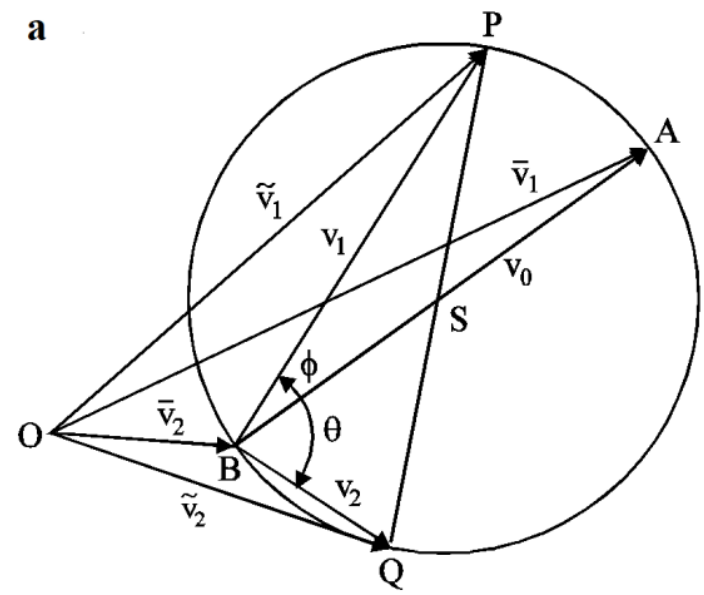

b

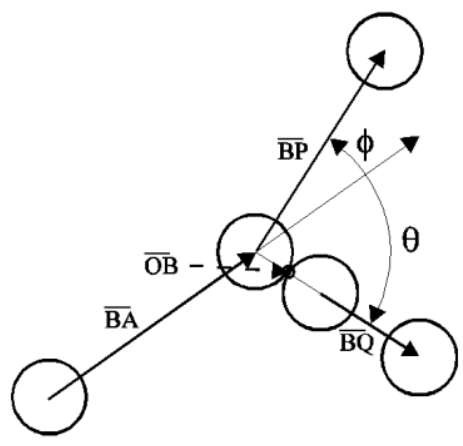

Figure 3. Solution $\mathrm{P}$, Q located on a sphere surface

Then for fixed magnitudes of $v_{j}$ and $v_{k}$, if $v_{k}$ is fixed in the horizontal direction but changed the direction of $v_{j}$, then point A will be located on a spherical surface as shown in Fig. 4. And the probability of point A on the surface is uniformly distributed since $v_{j}$ has equal opportunity in any direction. The center of the surface $\mathrm{A}$ is at point $\mathrm{O}$ and its radius is $v_{j} . \mathrm{S}$ is the middle point between $\mathrm{B}$ and $\mathrm{A}$ and is the center of the sphere surface $\mathrm{P}$ (also in Fig. 2 and 3). The point $\mathrm{S}$ will be located on a smaller sphere surface center at $\mathrm{C}$ (middle point of $\mathrm{O}$ and $\mathrm{B}$ ) with radius $v_{j} / 2$. Since $\overline{C S}$ is always parallel to $\overline{O A}$, the point $\mathrm{S}$ on the sphere surface $\mathrm{S}$ is also uniformly distributed similarly to point A on the sphere surface A. Although the sphere surfaces $\mathrm{S}$ and A are fixed, the surface $\mathrm{P}$ is variable in center $S$ and radius $r_{2}$. 


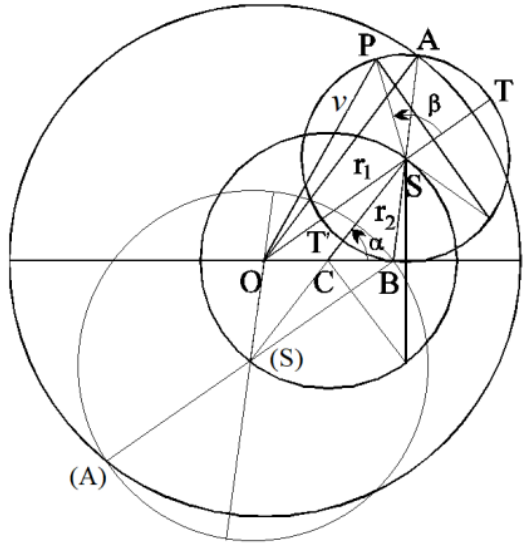

(T)

Figure 4. Three sphere surfaces A, S, P with centers at $\mathrm{O}, \mathrm{C}, \mathrm{S}$ with radius $v_{j}, v_{j} / 2, \mathrm{r}_{2}$

Next, the surface $\mathrm{S}$ and the surface $\mathrm{P}$ are used to find the probability of $v_{i}$. The location of $\mathrm{S}$ is defined by $\alpha$ (representing the relative moving direction before collision), and the location of $\mathrm{P}$ is defined by $\beta$ (representing the particle moving direction after collision).

1) The probability density of point $\mathrm{S}$ located on surface $\mathrm{S}$ at angle $\alpha$ is $P_{\alpha}(\alpha)=\frac{1}{2} \sin \alpha$.

2) The probability density of point $\mathrm{P}$ located on surface $\mathrm{P}$ at angle $\beta$ is $P_{\beta \mid \alpha}(\beta)=\frac{1}{2} \sin \beta$.

The $r_{1}$ and $r_{2}$ as shown in Fig. 4 can be computed from $\alpha$ as

$$
\begin{aligned}
& r_{1}\left(\alpha ; v_{j}, v_{k}\right)=\overline{O S}=\frac{1}{2} \sqrt{\left(v_{j} \cos \alpha+v_{k}\right)^{2}+\left(v_{j} \sin \alpha\right)^{2}}=\frac{1}{2} \sqrt{v_{j}^{2}+v_{k}^{2}+2 v_{j} v_{k} \cos \alpha} \\
& r_{2}\left(\alpha ; v_{j}, v_{k}\right)=\overline{S B}=\frac{1}{2} \sqrt{\left(v_{j} \cos \alpha-v_{k}\right)^{2}+\left(v_{j} \sin \alpha\right)^{2}}=\frac{1}{2} \sqrt{v_{j}^{2}+v_{k}^{2}-2 v_{j} v_{k} \cos \alpha}
\end{aligned}
$$

So the relation between $v$ and $\beta$ for fixed $r_{1}$ and $r_{2}$ is

Hence

$$
v\left(\beta ; r_{1}, r_{2}\right)=\overline{O P}=\sqrt{\left(r_{2} \cos \beta+r_{1}\right)^{2}+\left(r_{2} \sin \beta\right)^{2}}=\sqrt{r_{1}^{2}+r_{2}^{2}+2 r_{1} r_{2} \cos \beta}
$$

$$
\frac{d v}{d \beta}=\frac{-r_{1} r_{2} \sin \beta}{\sqrt{r_{1}^{2}+r_{2}^{2}+2 r_{1} r_{2} \cos \beta}}=\frac{-r_{1} r_{2} \sin \beta}{v}
$$

Using the relation above to change $P_{\beta \mid \alpha}(\beta)=\frac{1}{2} \sin \beta$ to

$$
P_{v \mid \alpha}(v)=P_{\beta \mid \alpha}(\beta)\left|\frac{d \beta}{d v}\right|=\frac{v}{2 r_{1} r_{2}}
$$

moreover, consider all density for $\alpha$ to get (Note 3)

$$
\begin{gathered}
\psi\left(v ; v_{j}, v_{k}\right)=\int_{\alpha_{\min }}^{\pi-\alpha_{\min }} P_{v \mid \alpha}(v) P_{\alpha}(\alpha) d \alpha=\int_{\alpha_{\min }}^{\pi-\alpha_{\min }} \frac{v}{4 r_{1} r_{2}} \sin \alpha d \alpha \\
=\int_{\alpha_{\min }}^{\pi-\alpha_{\min }} \frac{v \sin \alpha d \alpha}{\sqrt{\left(v_{j}^{2}+v_{k}^{2}\right)^{2}-4 v_{j}^{2} v_{k}^{2} \cos ^{2} \alpha}}=\frac{v}{v_{j} v_{k}} \sin ^{-1}\left(\frac{2 v_{j} v_{k}}{v_{j}^{2}+v_{k}^{2}} \cos \alpha_{\min }\right)
\end{gathered}
$$

where $\alpha_{\text {min }}=0$ for $v_{k} \leq v \leq v_{j}$, else $\alpha_{\text {min }}$ are where $v=\overline{O T}$ or $v=\overline{O T^{\prime}}$ (Fig. 4) as follows

$$
v=\left|r_{1} \pm r_{2}\right|=\frac{1}{2}\left|\sqrt{v_{j}^{2}+v_{k}^{2}+2 v_{j} v_{k} \cos \alpha_{\min }} \pm \sqrt{v_{j}^{2}+v_{k}^{2}-2 v_{j} v_{k} \cos \alpha_{\min }}\right|
$$

Both equations have identical solutions for $\cos \alpha_{\min }$ as (Note 4)

$$
\cos \alpha_{\min }=\frac{v}{v_{j} v_{k}} \sqrt{v_{j}^{2}+v_{k}^{2}-v^{2}}
$$

Substitution of Eq.(20) into Eq.(18) yields the probability density function as (Regions are shown in Fig. 6) 


$$
\begin{array}{rlrl}
\psi\left(v ; v_{j}, v_{k}\right) & =\frac{v}{v_{j} v_{k}} \sin ^{-1}\left(\frac{2 v}{v_{j}^{2}+v_{k}^{2}} \sqrt{v_{j}^{2}+v_{k}^{2}-v^{2}}\right), 0 \leq v \leq v_{k}\left(\operatorname{region} A_{2}\right) \\
& =\frac{v}{v_{j} v_{k}} \sin ^{-1}\left(\frac{2 v_{j} v_{k}}{v_{j}^{2}+v_{k}^{2}}\right), & & 0 \leq v_{k} \leq v \leq v_{j} \quad\left(\operatorname{regions} B_{2} \text { and } B_{1}\right) \\
& =0, & & v \geq \sqrt{v_{j}^{2}+v_{k}^{2}}\left(\operatorname{region} B_{0}\right)
\end{array}
$$

\section{Numerical Iteration}

It is easy to do the iteration by 65 equal spaced discrete speeds beginning from $\mathrm{v}_{1}=0.5$ in increments of 1.0 and end up to $\mathrm{v}_{65}=64.5$, where speeds over 64.5 are truncated, and therefore the probabilities are assumed to be zero. For discrete speeds, the integration changes to summation as follows $\left(\Delta v_{k} \Delta v_{j}=1\right)$

$$
\mathrm{P}_{\text {new }}\left(v_{i}\right)=\sum_{j=1}^{65} \sum_{k=1}^{65} \psi\left(v_{i} ; v_{j}, v_{k}\right) \mathrm{P}_{\text {old }}\left(v_{j}\right) \mathrm{P}_{\text {old }}\left(v_{k}\right), \quad i=1,2,3, \ldots, 65
$$

If we assume the Root-Mean-Square speed is 16.5 , and the initial speeds of all particles are 16.5, that is $\mathrm{P}_{\text {old }}\left(v_{17}\right)=$ 1 and all others $\mathrm{P}_{\text {old }}\left(v_{i}\right)=0$, for $i \neq 17$. Use the equation above to get $\mathrm{P}_{\text {new }}\left(v_{i}\right)$, and set $\mathrm{P}_{\text {old }}\left(v_{i}\right)=\mathrm{P}_{\text {new }}\left(v_{i}\right)$ for next iteration. After nine iterations, the distribution curves converge to the Maxwell speed distribution as shown in Figure 5.

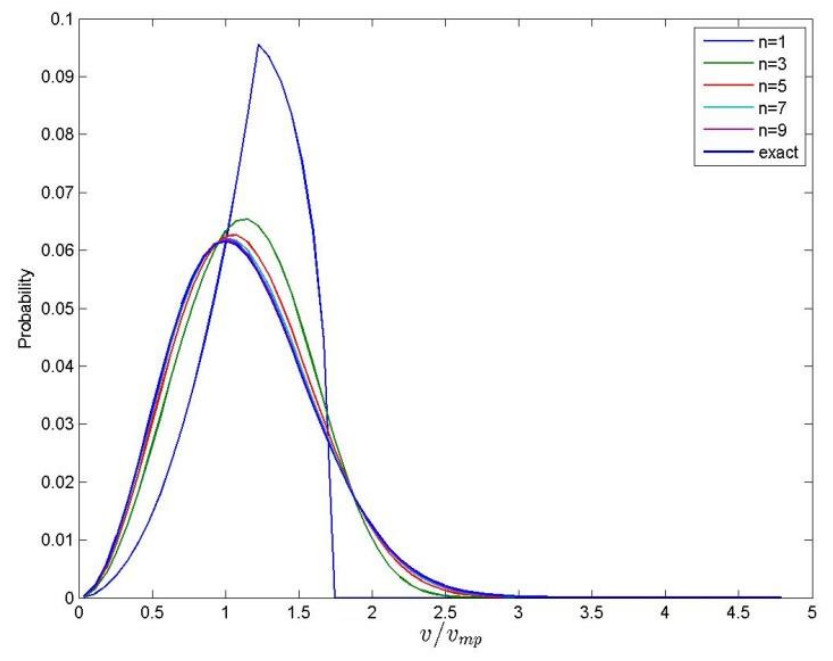

Figure 5. Nine iterations converge to the Maxwell speed distribution

As shown in Fig. 5, the horizontal axis for the speed, $v$, has been normalized by the most probable speed, $v_{m p}$. Therefore the peak dirtribution density is just at $v / v_{m p}=h v=1$ as we would expect. Where $v_{m p}=\sqrt{2 / 3} v_{r m s}=$ 13.47 , and the peak distribution density is $(4 h / \sqrt{\pi}) e^{-1}=0.0616$. Any initial distribution may be assumed, as long as the initial RMS speed less than $25 \%$ of the maximum speed used, i.e., 64.5 in the presented case, the distribution curve always converges to the Maxwell speed distribution.

\section{Analytical Integration}

Let $P_{\text {old }}$ be the Maxwell speed distribution $P(v)=\frac{4 h^{3}}{\sqrt{\pi}} v^{2} e^{-h^{2} v^{2}}$ and compute $P_{\text {new }}$ from the following equation with four regions as shown in Fig. 6.

$$
\begin{aligned}
P_{\text {new }}(v) & =\int_{0}^{\infty} \int_{0}^{\infty} \psi\left(v ; v_{j}, v_{k}\right) P_{\text {old }}\left(v_{j}\right) P_{\text {old }}\left(v_{k}\right) d v_{j} d v_{k} \\
& =\frac{32 h^{6}}{\pi} \iint_{A_{1}+A_{2}} v v_{j} v_{k} \sin ^{-1}\left(\frac{2 v}{v_{j}^{2}+v_{k}^{2}} \sqrt{v_{j}^{2}+v_{k}^{2}-v^{2}}\right) e^{-h^{2}\left(v_{j}^{2}+v_{k}^{2}\right)} d v_{j} d v_{k}
\end{aligned}
$$




$$
+\frac{32 h^{6}}{\pi} \iint_{B_{1}+B_{2}} v v_{j} v_{k} \sin ^{-1}\left(\frac{2 v_{j} v_{k}}{v_{j}^{2}+v_{k}^{2}}\right) e^{-h^{2}\left(v_{j}^{2}+v_{k}^{2}\right)} d v_{j} d v_{k}
$$

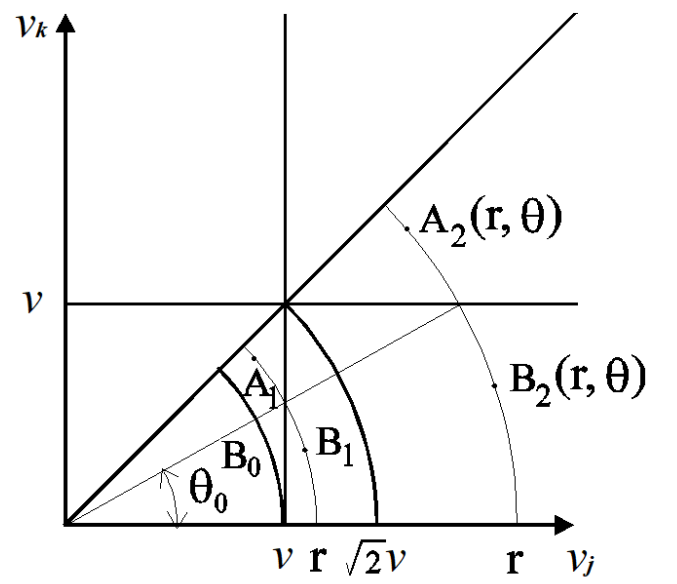

Figure 6. Five regions for integrations (for fixed $v$ )

Change rectangular coordinates to polar coordinates: $v_{j}=r \cos \theta, v_{k}=r \sin \theta$

$$
\begin{gathered}
P_{\text {new }}(v)=\frac{32 h^{6} v}{\pi} \iint_{A_{1}+A_{2}} r^{2} \sin \theta \cos \theta \sin ^{-1}\left(\frac{2 v}{r} \sqrt{1-\left(\frac{v}{r}\right)^{2}}\right) e^{-h^{2} r^{2}} r d r d \theta \\
+\frac{32 h^{6} v}{\pi} \iint_{B_{1}+B_{2}} r^{2} \sin \theta \cos \theta \sin ^{-1}(2 \sin \theta \cos \theta) e^{-h^{2} r^{2}} r d r d \theta
\end{gathered}
$$

In Fig. 6, $\theta_{0}=\cos ^{-1} \frac{v}{r}$ is the angle at the boundary of regions $A_{1}$ and $B_{1}$, and $\theta_{0}=\sin ^{-1} \frac{v}{r}$ when in regions $A_{2}$ and $B_{2}$. And since $\sin ^{-1}\left(\frac{2 v}{r} \sqrt{1-\left(\frac{v}{r}\right)^{2}}\right)=2 \theta_{0}$, we have

$$
\begin{aligned}
P_{\text {new }}(v) & =\frac{16 h^{6} v}{\pi} \int_{v}^{\infty}\left\{\int_{\theta_{0}}^{\pi / 4} \sin 2 \theta\left(2 \theta_{0}\right) d \theta+\int_{0}^{\theta_{0}} \sin 2 \theta(2 \theta) d \theta\right\} e^{-h^{2} r^{2}} r^{3} d r \\
& =\frac{8 h^{6} v}{\pi} \int_{v}^{\infty}\left\{2 \theta_{0}[-\cos 2 \theta]_{\theta_{0}}^{\pi / 4}+[-2 \theta \cos 2 \theta+\sin 2 \theta]_{0}^{\theta_{0}}\right\} e^{-h^{2} r^{2}} r^{3} d r \\
& =\frac{8 h^{6} v}{\pi} \int_{v}^{\infty}\left\{2 \theta_{0} \cos 2 \theta_{0}-2 \theta_{0} \cos 2 \theta_{0}+\sin 2 \theta_{0}\right\} e^{-h^{2} r^{2}} r^{3} d r \\
& =\frac{8 h^{6} v}{\pi} \int_{v}^{\infty} \sin 2 \theta_{0} e^{-h^{2} r^{2}} r^{3} d r=\frac{8 h^{6} v}{\pi} \int_{v}^{\infty} \frac{2 v}{r} \sqrt{1-\left(\frac{v}{r}\right)^{2}} e^{-h^{2} r^{2}} r^{3} d r \\
& =\frac{16 h^{6} v^{2}}{\pi} \int_{v}^{\infty} \sqrt{r^{2}-v^{2}} e^{-h^{2} r^{2}} r d r
\end{aligned}
$$

Change variable by $u^{2}=r^{2}-v^{2}, 2 u d u=2 r d r$, and use $\int_{0}^{\infty} P_{u}(u) d u=\frac{4 h^{3}}{\sqrt{\pi}} \int_{0}^{\infty} u^{2} e^{-h^{2} u^{2}} d u=1$ to get

$$
P_{\text {new }}(v)=\frac{16 h^{6} v^{2}}{\pi} \int_{0}^{\infty} u e^{-h^{2}\left(v^{2}+u^{2}\right)} u d u=\frac{4 h^{3}}{\sqrt{\pi}} v^{2} e^{-h^{2} v^{2}}\left[\frac{4 h^{3}}{\sqrt{\pi}} \int_{0}^{\infty} u^{2} e^{-h^{2} u^{2}} d u\right]=\frac{4 h^{3}}{\sqrt{\pi}} v^{2} e^{-h^{2} v^{2}}
$$

The analytical integration result of $P_{n e w}(v)$ is just the Maxwell speed distribution as we would expect. This concludes the proof that the Maxwell speed distribution is correct from the random collisions of the particles.

\section{Conclusions and Further Studies}

It is not only interesting but also very important to get the function $\psi\left(v ; v_{j}, v_{k}\right)$ since this is from which the Maxwell speed distribution can be proved. From the derivation of the function $\psi\left(v ; v_{j}, v_{k}\right)$, we can reveal the basic mechanism behind the macroscopic phenomenon. The mechanics of the collision of particles is a bridge between microscopic 
behavior and macroscopic phenomenon.

This paper only investigates the collisions of equal mass particles. Further study may be on the collisions of unequal mass particles and may be used to give a mechanical proof of Avogadro's law. The procedures of this paper may also be used for the collisions of charged particles.

\section{Acknowledgments}

We are grateful to Dr. Steven K. Lang, Applications Engineering Director of Intel Microelectronics Asia Ltd. Taiwan Branch, for his valuable suggestions. We also thanks to the reviewers for their valuable suggestions.

\section{References}

Boltzmann, L. (1872). Weitere studien uber das Warmegleichgewicht unter Gasmolekulen, Sitzungsbenchte der Kaiserlichen Akademie der Wissenschaften in Wien, Mathematisch-Naturwissenschaftliche Classe, 66, $275-370$.

Brush, S. G. (1966). Kinetic theory Vol.2. Irreversible processes. Pergamon Press, ISBN 9781483155937 1483155935.Selection.2:Ludwig Boltzmann:Further Studies on the Thermal Equilibrium of Gas Molecules (from Sitzungsbenchte der Kaiserlichen Akademie der Wissenschaften,Vienna, 1872), 88-175.

Garrod, C. (1995). Statistical mechanics and thermodynamics. University of California, Davis, New York Oxford: Oxford University Press, ISBN 0-19-508523-X (IBM software).

Landau L. D., \& Lifshitz, E. M. (1969). Statistical physics. Institute of Physical Problems, U.S.S.R. Academy of Science, Vol.5 of Course of Theoretical Physic, Translated from the Russian by Peierls, E. and Peierls, R.F., London, Paris: Pergamon Press, ISBN 0-201-0-04167-7.

Maudlin, T. (2013). How to derive the equilibrium velocity distribution two ways (Neither of which is what you are expecting). Cosmology Summer School, Santa Cruz, July 26, 2013, NYU, Retrieved from http://hipacc.ucsc.edu/IPC2013/slides/130626_TimMaudlin.pdf

Maxwell, J. C. (1860a). V. Illustrations of the dynamical theory of gases, Part I. On the motions and collisions of perfectly elastic spheres, The London, Edinburgh, and Dubin Philosophical Magazine and Journal of Science, $4^{\text {th }}$ Series, 19, 19-32. https://doi.org/10.1080/14786446008642818

Maxwell, J. C. (1860b). II. Illustrations of the dynamical theory of gases, Part II. On the process of diffusion of two or more kinds of moving particles among one another, The London, Edinburgh, and Dubin Philosophical Magazine and Journal of Science, $4^{\text {th }}$ Series, 20, 21-37. https://doi.org/10.1080/14786446008642902

Maxwell, J. C. (1868). XV. On the dynamical theory of gases, The London, Edinburgh, and Dubin Philosophical Magazine and Journal of Science, $4^{\text {th }}$ Series, 35, 129-145, 185-217. https://doi.org/10.1080/14786446808639951, https://doi.org/10.1080/14786446808639963

McQuarrie, D. A. (1976). Statistical mechanics. Indiana University, Harper Collins Publishers, ISBN 06-044366-9.

\section{Notes}

Note 1.

Begin from following equations

$$
\begin{array}{r}
\mathrm{M}_{1} \mathrm{v}_{0}=\mathrm{M}_{2} \mathrm{v}_{2} \cos \theta+\mathrm{M}_{1} \mathrm{v}_{1} \cos \phi \\
0=\mathrm{M}_{2} \mathrm{v}_{2} \sin \theta-\mathrm{M}_{1} \mathrm{v}_{1} \sin \phi \\
\mathrm{M}_{1} \mathrm{v}_{0}^{2} / 2=\mathrm{M}_{2} \mathrm{v}_{2}^{2} / 2+\mathrm{M}_{1} \mathrm{v}_{1}^{2} / 2
\end{array}
$$

Regroup and take the square of Eqs.(N1) and (N2) to get

$$
\begin{aligned}
& \mathrm{M}_{1}^{2} \mathrm{v}_{1}^{2} \cos ^{2} \phi=\left(\mathrm{M}_{2} \mathrm{v}_{2} \cos \theta-\mathrm{M}_{1} \mathrm{v}_{0}\right)^{2} \\
& =\mathrm{M}_{2}^{2} \mathrm{v}_{2}^{2} \cos ^{2} \theta+\mathrm{M}_{1}^{2} \mathrm{v}_{0}^{2}-2 \mathrm{M}_{1} \mathrm{M}_{2} \mathrm{v}_{0} \mathrm{v}_{2} \cos \theta \\
& \mathrm{M}_{1}^{2} \mathrm{v}_{1}^{2} \sin ^{2} \phi=\mathrm{M}_{2}^{2} \mathrm{v}_{2}^{2} \sin ^{2} \theta
\end{aligned}
$$

Add Eqs.(N4) and (N5) to get Eq.(N6), multiply Eq.(N3) by $M_{1}$ to get Eq.(N7), and then subtract Eq.(N6) from Eq.(N7) to get Eq.(N8).

$$
\begin{gathered}
M_{1}^{2} v_{1}^{2}-M_{2}^{2} v_{2}^{2}=M_{1}^{2} v_{0}^{2}-2 M_{1} v_{0} M_{2} v_{2} \cos \theta \\
M_{1}^{2} v_{1}^{2}+M_{1} M_{2} v_{2}^{2}=M_{1}^{2} v_{0}^{2} \\
\left(M_{1} M_{2}+M_{2}^{2}\right) v_{2}^{2}=2 M_{1} v_{0} M_{2} v_{2} \cos \theta
\end{gathered}
$$

From Eq.(N8) we get $v_{2}$ also, substitute to Eq.(N3) to get $v_{1}$ as 


$$
\begin{gathered}
\mathrm{v}_{2}=\mathrm{v}_{0}\left(\frac{2 \mathrm{M}_{1}}{\mathrm{M}_{1}+\mathrm{M}_{2}}\right) \cos \theta \\
\mathrm{v}_{1}=\mathrm{v}_{0} \sqrt{1-\frac{4 \mathrm{M}_{1} \mathrm{M}_{2}}{\left(\mathrm{M}_{1}+\mathrm{M}_{2}\right)^{2}} \cos ^{2} \theta}
\end{gathered}
$$

Next, we will find the solution for $\phi$ : multiply Eq.(N1) \& (N2) by $\sin \phi \& \cos \phi$ and add together to get

$$
\mathrm{M}_{1} \mathrm{v}_{0} \sin \phi=\mathrm{M}_{2} \mathrm{v}_{2}(\cos \theta \sin \phi+\sin \theta \cos \phi)
$$

Since $\cos \theta \sin \phi+\sin \theta \cos \phi=\sin (\theta+\phi)$, we get

$$
\sin (\theta+\phi)=\frac{\mathrm{M}_{1} \mathrm{v}_{0}}{\mathrm{M}_{2} \mathrm{v}_{2}} \sin \phi
$$

From Eq.(N2) to get Eq.(N13). Substitute to Eq.(N12) and by Eq.(N10) to get

$$
\begin{gathered}
\sin \phi=\frac{\mathrm{M}_{2} \mathrm{v}_{2}}{\mathrm{M}_{1} \mathrm{v}_{1}} \sin \theta \\
\sin (\theta+\phi)=\frac{\mathrm{v}_{0}}{\mathrm{v}_{1}} \sin \theta=\frac{\sin \theta}{\sqrt{1-\frac{4 \mathrm{M}_{1} \mathrm{M}_{2}}{\left(\mathrm{M}_{1}+\mathrm{M}_{2}\right)^{2}} \cos ^{2} \theta}}
\end{gathered}
$$

For $\mathrm{M}_{1}=\mathrm{M}_{2}$, from Eqs.(N9)(N10)(N14), we get the solutions as

$$
\begin{aligned}
\mathrm{v}_{2} & =\mathrm{v}_{0} \cos \theta \\
\mathrm{v}_{1} & =\mathrm{v}_{0} \sin \theta \\
\sin (\theta+\phi) & =1 \quad \text { or } \quad \phi=\frac{\pi}{2}-\theta
\end{aligned}
$$

Note 2.

$\mathrm{P}$ and $\mathrm{Q}$ are always located on the sphere surface, and the probability is uniform on this surface. Since the probability of the point inside the circle in Fig. 2(b) (radius=diameter of particle $=D$ ) is uniformly distributed. The reasons are based on the following factors:

1) The area of the ring on the sphere surface (Fig. 2(a)) is $2 \pi\left(\frac{v_{0}}{2} \sin 2 \theta\right)\left(\frac{v_{0}}{2} 2 d \theta\right)=2 \pi v_{0}^{2} \sin \theta \cos \theta d \theta$.

2) The area of the ring inside the circular plane disk (Fig. 2(b)) is $2 \pi(\mathrm{D} \sin \theta) d(\mathrm{D} \sin \theta)=2 \pi D^{2} \sin \theta \cos \theta d \theta$

3) The ratio of the two areas is $\mathrm{v}_{0}^{2} / D^{2}$, it is not dependent on $\theta$.

4) When the center of the particle hits inside the ring of the disk, the Q point must locate inside the ring on the sphere surface.

5) It has equal opportunity to hit on any point inside the circle.

Note 3.

$$
\begin{aligned}
& \psi\left(v ; v_{j}, v_{k}\right)=\int_{\alpha_{\min }}^{\alpha_{\max }} P_{v \mid \alpha}(v) P_{\alpha}(\alpha) d \alpha=\int_{\alpha_{\min }}^{\pi-\alpha_{\min }} \frac{v}{4 r_{1} r_{2}} \sin \alpha d \alpha \\
& =\int_{\alpha_{\min }}^{\pi-\alpha_{\min }} \frac{v \sin \alpha d \alpha}{\sqrt{\left(v_{j}^{2}+v_{k}^{2}\right)^{2}-4 v_{j}^{2} v_{k}^{2} \cos ^{2} \alpha}}=\frac{v}{2 v_{j} v_{k}} \int_{\alpha_{\min }}^{\pi-\alpha_{\min }} \frac{\frac{2 v_{j} v_{k}}{v_{j}^{2}+v_{k}^{2}} \sin \alpha d \alpha}{\sqrt{1-\left(\frac{2 v_{j} v_{k}}{v_{j}^{2}+v_{k}^{2}} \cos \alpha\right)^{2}}}
\end{aligned}
$$

Change variable by $\sin u=\frac{2 v_{j} v_{k}}{v_{j}^{2}+v_{k}^{2}} \cos \alpha, \cos u d u=-\frac{2 v_{j} v_{k}}{v_{j}^{2}+v_{k}^{2}} \sin \alpha d \alpha$, to get

$$
\begin{aligned}
\psi\left(v ; v_{j}, v_{k}\right) & =\frac{-v}{2 v_{j} v_{k}} \int_{u_{\min }}^{u_{\max }} \frac{\cos u d u}{\sqrt{1-\sin ^{2} u}}=\frac{-v}{2 v_{j} v_{k}} \int_{u_{\min }}^{u_{\max }} \frac{\cos u d u}{\cos u}=\frac{v}{2 v_{j} v_{k}}\left(u_{\min }-u_{\max }\right) \\
& =\frac{v}{2 v_{j} v_{k}}\left(\sin ^{-1}\left(\frac{2 v_{j} v_{k}}{v_{j}^{2}+v_{k}^{2}} \cos \alpha_{\min }\right)-\sin ^{-1}\left(\frac{2 v_{j} v_{k}}{v_{j}^{2}+v_{k}^{2}} \cos \left(\pi-\alpha_{\min }\right)\right)\right)
\end{aligned}
$$




$$
=\frac{v}{v_{j} v_{k}} \sin ^{-1}\left(\frac{2 v_{j} v_{k}}{v_{j}^{2}+v_{k}^{2}} \cos \alpha_{\min }\right)
$$

Note 4.

Begin from the equation

$$
v=\left|r_{1} \pm r_{2}\right|=\frac{1}{2}\left|\sqrt{v_{j}^{2}+v_{k}^{2}+2 v_{j} v_{k} \cos \alpha_{\min }} \pm \sqrt{v_{j}^{2}+v_{k}^{2}-2 v_{j} v_{k} \cos \alpha_{\min }}\right|
$$

Square to get

$$
4 v^{2}=v_{j}^{2}+v_{k}^{2}+2 v_{j} v_{k} \cos \alpha_{\text {min }}+v_{j}^{2}+v_{k}^{2}-2 v_{j} v_{k} \cos \alpha_{\min } \pm 2 \sqrt{\left(v_{j}^{2}+v_{k}^{2}\right)^{2}-\left(2 v_{j} v_{k} \cos \alpha_{\text {min }}\right)^{2}}
$$

Square again to get

$$
\left(v_{j}^{2}+v_{k}^{2}\right)^{2}-\left(2 v_{j} v_{k} \cos \alpha_{\text {min }}\right)^{2}=\left(v_{j}^{2}+v_{k}^{2}-2 v^{2}\right)^{2}=\left(v_{j}^{2}+v_{k}^{2}\right)^{2}-4 v^{2}\left(v_{j}^{2}+v_{k}^{2}\right)+4 v^{4}
$$

or

$$
\left(2 v_{j} v_{k} \cos \alpha_{\min }\right)^{2}=4 v^{2}\left(v_{j}^{2}+v_{k}^{2}-v^{2}\right)
$$

Hence

$$
\cos \alpha_{\min }= \pm \frac{v}{v_{j} v_{k}} \sqrt{v_{j}^{2}+v_{k}^{2}-v^{2}}
$$

Therefore we have

$$
\begin{gathered}
\cos \alpha_{\text {min }}=\frac{v}{v_{j} v_{k}} \sqrt{v_{j}^{2}+v_{k}^{2}-v^{2}} \\
\cos \alpha_{\text {max }}=\cos \left(\pi-\alpha_{\text {min }}\right)=-\frac{v}{v_{j} v_{k}} \sqrt{v_{j}^{2}+v_{k}^{2}-v^{2}}
\end{gathered}
$$

\section{Copyrights}

Copyright for this article is retained by the author(s), with first publication rights granted to the journal.

This is an open-access article distributed under the terms and conditions of the Creative Commons Attribution license (http://creativecommons.org/licenses/by/4.0/). 